\title{
Some Examples of Post-Measurement Nonlocal Gates
}

\author{
D. SONG* \\ School of Liberal Arts, Korea University of Technology \& Education, Chungnam 330-708, Korea
}

(Received August 30, 2011)

\begin{abstract}
Several proposed quantum computer models include measurement processes, in order to implement nonlocal gates and create necessary entanglement resources during the computation. We discuss some examples in which the measurements can be delayed for two- and three-qubit nonlocal gates. We also discuss implementing arbitrary nonlocal gates when measurements are included during the process.
\end{abstract}

PACS: 03.67.Lx, 03.65.Ud

\section{Introduction}

Various people have introduced [1-3] nonlocal gates so that when entanglement resources are provided, one can perform multiple qubit gates on widely separated qubits using only local operations. Using the concept of an entanglement bus, a quantum computer architecture has been proposed [4] to efficiently implement nonlocal gates on a lattice model, allowing only nearest neighbor interactions. However, these nonlocal gates involve measurement processes during the implementation of the gates. If they were to be used during a large-scale quantum computation, a large number of measurements would be required. This will be particularly difficult for systems where measurement time is substantially longer than unitary operation time or the case when measurement errors are greater than errors due to unitary operations. It is therefore desirable to seek a possibility such that all the measurements can be delayed until the end of the computation. In this paper, we introduce some examples in which measurements can be delayed until the end of the implementation of two- and three-qubit nonlocal gates.

Eisert et al. [1] (also in [2]) have discussed the use of classical and entanglement resources for the implementation of nonlocal gates. Here, we would like to consider implementing nonlocal gates on a quantum computer. Since we have a rather reliable classical information processor, we will be concerned mainly with entanglement resources rather than classical information. Firstly, we will review the implementation of nonlocal gates with measurements in the middle of the process. Arbitrary quantum gates on two distant qubits can be performed with two EPR pairs, or ebits, by using teleportation.

\footnotetext{
* e-mail: dsong@kut.ac.kr
}

\section{Some nonlocal gates}

Let us take an example to see how it works. As shown in Fig. 1, given two distant localized states $(a|0\rangle+b|1\rangle)_{A}$ and $(c|0\rangle+d|1\rangle)_{B}$, we want to perform nonlocal gates between $A$ and $B$ using entanglement resources, in this case, we want to apply CNOT with the control qubit $A$ and target $B$ followed by another CNOT with the control $B$ and target $A$. We start by teleporting qubit $A$ using an ebit of $A_{1}$ and $B_{1}$,

$$
(a|0\rangle+b|1\rangle)_{A}(|00\rangle+|11\rangle)_{A_{1} B_{1}}
$$

by making a Bell measurement on $A$ and $A_{1}$. We will omit the normalization factor when the coefficients are equal. The Bell measurement can be performed by applying CNOT followed by the Hadamard gate which transforms, $\left|\phi^{+}\right\rangle \equiv|00\rangle+|11\rangle \rightarrow|00\rangle,\left|\psi^{+}\right\rangle \equiv|01\rangle+|10\rangle \rightarrow$ $|01\rangle,\left|\psi^{-}\right\rangle \equiv|01\rangle-|10\rangle \rightarrow|11\rangle$ and $\left|\phi^{-}\right\rangle \equiv|00\rangle-$ $|11\rangle \rightarrow|10\rangle$. But we will just use the original Bell basis, $\left|\phi^{ \pm}\right\rangle,\left|\psi^{ \pm}\right\rangle$, for simplicity. Depending on the Bell measurement result on $A A_{1}$, the following correction gates are applied to $B_{1},\left|\phi^{+}\right\rangle \rightarrow \mathbf{1},\left|\psi^{+}\right\rangle \rightarrow \sigma_{x},\left|\psi^{-}\right\rangle \rightarrow \sigma_{z} \sigma_{x}$, and $\left|\phi^{-}\right\rangle \rightarrow \sigma_{z}$, yielding the result $(a|0\rangle+b|1\rangle)_{B_{1}}$. Next we perform the desired arbitrary local operation on $B_{1}$ and $B$, i.e. $U_{2} \equiv \mathrm{CNOT}_{B B_{1}} \mathrm{CNOT}_{B_{1} B}$. The result becomes,

$$
(a c|00\rangle+a d|11\rangle+b c|01\rangle+b d|10\rangle)_{B_{1} B} .
$$

We then use another ebit $(|00\rangle+|11\rangle)_{A_{2} B_{2}}$ and perform the Bell measurement on $B_{1}$ and $B_{2}$. As before, depending on the results, we apply the same correction gates to $A_{2}$. Finally swapping $A_{2}$ with $A$ yields the final state we want as in (2) for qubits $A$ and $B$. It is clear that this procedure can be generalized to arbitrary two-qubit operations for $U_{2}$.

In a similar manner, this can be generalized to $n$ qubit arbitrary nonlocal gates with $2(n-1)$ ebits. We use $(n-1)$ ebits to teleport $(n-1)$ of the qubits near to the 


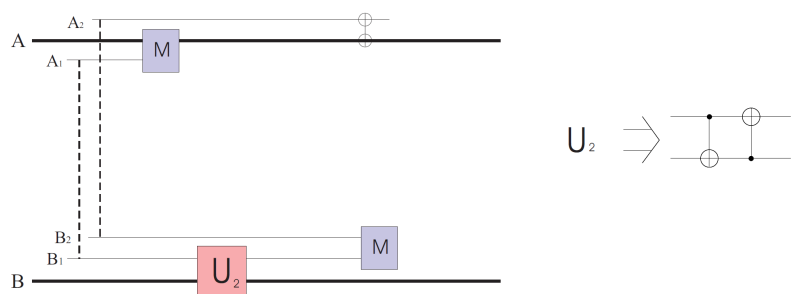

Fig. 1. Arbitrary nonlocal two-qubit gate, $U_{2}$, between $A$ and $B$ using teleportation. Using ebit $A_{1} B_{1}, A$ is teleported to $B_{1}$. The desired two-qubit gate is performed on $B_{1} B$, we then teleport $B_{1}$ back using ebit $A_{2} B_{2}$ and swap $A_{2}$ with $A$. $M$ refers to the Bell measurement.

$n$-th qubit, and then perform the desired gates on them and teleport each back using another $(n-1)$ ebits.

So far, we've considered the nonlocal gates where measurements were involved in the middle of the process as in previous studies $[1,2]$. In the following, we introduce two-qubit and three-qubit nonlocal gates where measurements may be delayed until the end of the gate. We first consider a nonlocal CNOT gate on qubits $A$ and $B$ using the ebit $A_{1} B_{1}$ as follows:

$$
(a|0\rangle+b|1\rangle)_{A}(|00\rangle+|11\rangle)_{A_{1} B_{1}}(c|0\rangle+d|1\rangle)_{B} .
$$

This can be re-written as

$$
\begin{aligned}
& \left\{\left|\phi^{+}\right\rangle_{A A_{1}}(a|0\rangle+b|1\rangle)_{B_{1}}+\left|\psi^{+}\right\rangle_{A A_{1}}(a|1\rangle+b|0\rangle)_{B_{1}}\right. \\
& \left.+\left|\psi^{-}\right\rangle_{A A_{1}}(a|1\rangle-b|0\rangle)_{B_{1}}+\left|\phi^{-}\right\rangle_{A A_{1}}(a|0\rangle-b|1\rangle)_{B_{1}}\right\} \\
& \quad \times(c|0\rangle+d|1\rangle)_{B} .
\end{aligned}
$$

We start by applying the desired CNOT gate on $B_{1}$ and $B$. Next we use the second ebit $(|00\rangle+|11\rangle)_{A_{2} B_{2}}$ as shown in Fig. 2 and swap $A$ with $A_{2}$. It now remains to perform two local Bell measurements on $B_{1} B_{2}$ and $A_{1} A_{2}$. The result on $B_{1} B_{2}$ will correct qubit $A$ as $\left|\phi^{+}\right\rangle \rightarrow \mathbf{1}$, $\left|\psi^{+}\right\rangle \rightarrow \sigma_{x},\left|\psi^{-}\right\rangle \rightarrow \sigma_{z} \sigma_{x}$, and $\left|\phi^{-}\right\rangle \rightarrow \sigma_{z}$. Next, the result on $A_{1} A_{2}$ will correct both qubits $A$ and $B$ as follows: $\phi^{+} \rightarrow \mathbf{1}^{A} \otimes \mathbf{1}^{B}, \psi^{+} \rightarrow \sigma_{x}^{A} \otimes \sigma_{x}^{B}, \psi^{-} \rightarrow\left(\sigma_{z} \sigma_{x}\right)^{A} \otimes \sigma_{x}^{B}$, $\phi^{-} \rightarrow \sigma_{z}^{A} \otimes \mathbf{1}^{B}$.
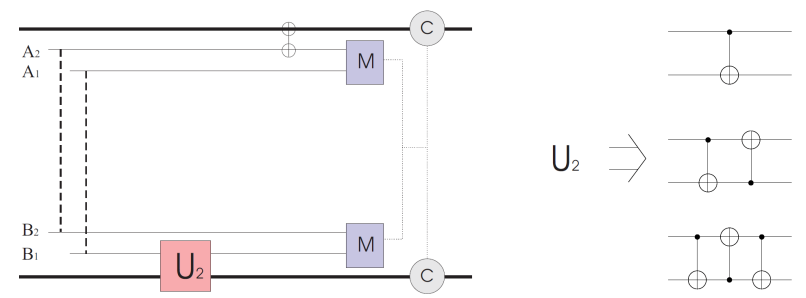

Fig. 2. Post-measurement two-qubit nonlocal gate for one, two and three CNOT's. Using two ebits $A_{1} B_{1}$ and $A_{2} B_{2}$, two-qubit gates are performed on $B_{1}$ and $B$ and $A_{2}$ and $A$ are swapped. The measurements are made at the end, and the correction gates dependent on $U_{2}$ are applied to $A$ and $B$.

This procedure can be generalized to other networks of CNOT gates. Let us consider a CNOT gate with control qubit $A$ and target $B$ followed by another CNOT with control $B$ and target $A$, i.e. $U_{2}=\mathrm{CNOT}_{B A} \mathrm{CNOT}_{A B}$. We follow the same network as in Fig. 2 and the result on $B_{1} B_{2}$ will correct just as in a single CNOT gate case. Then the result on $A_{1}$ and $A_{2}$ corrects again both $A$ and $B$ as follows: $\phi^{+} \rightarrow \mathbf{1}^{A} \otimes \mathbf{1}^{B}, \psi^{+} \rightarrow \mathbf{1}^{A} \otimes \sigma_{x}^{B}$, $\psi^{-} \rightarrow \sigma_{z}^{A} \otimes\left(\sigma_{z} \sigma_{x}\right)^{B}, \phi^{-} \rightarrow \sigma_{z}^{A} \otimes \sigma_{z}^{B}$. For three CNOT gates, i.e. a swap operation between $A$ and $B$, the correction gate for $A_{1} A_{2}$ is applied only to qubit $B$ as follows: $\phi^{+} \rightarrow \mathbf{1}, \psi^{+} \rightarrow \sigma_{x}^{B}, \psi^{-} \rightarrow\left(\sigma_{z} \sigma_{x}\right)^{B}, \phi^{-} \rightarrow \sigma_{z}^{B}$. Therefore, for a swap nonlocal gate, the correction gate for $A_{1} A_{2}$ is applied to only qubit $B$ while the correction gate for $B_{1} B_{2}$ is applied to $A$ as before.

Next we consider the case of operations on three qubits. Three arbitrary qubits $(a|0\rangle+b|1\rangle)_{A},(c|0\rangle+d|1\rangle)_{B}$, and $(e|0\rangle+f|1\rangle)_{C}$ with two ebits $A_{1} B_{1}$ and $B_{2} C_{2}$ can be written as

$$
\begin{aligned}
& \left\{\left|\phi^{+}\right\rangle(a|0\rangle+b|1\rangle)+\left|\psi^{+}\right\rangle(a|1\rangle+b|0\rangle)\right. \\
& \left.\left|\psi^{-}\right\rangle(a|1\rangle-b|0\rangle)+\left|\phi^{-}\right\rangle(a|0\rangle-b|1\rangle)\right\}_{A A_{1} B_{1}} \\
& \quad \otimes(c|0\rangle+d|1\rangle)_{B} \\
& \quad \otimes\left\{(e|0\rangle+f|1\rangle)\left|\phi^{+}\right\rangle+(e|1\rangle+f|0\rangle)\left|\psi^{+}\right\rangle\right. \\
& \left.\quad+(e|1\rangle-f|0\rangle)\left|\psi^{-}\right\rangle+(e|0\rangle-f|1\rangle)\left|\psi^{+}\right\rangle\right\}_{B_{2} C C_{2}}
\end{aligned}
$$

First we study the network $U_{3}=$ $\mathrm{CNOT}_{C A} \mathrm{CNOT}_{B C} \mathrm{CNOT}_{A B}$ as shown in Fig. 3. As in the two-qubit case, we apply these three CNOTs on $B_{1}, \quad B$ and $B_{2}$, i.e. $U_{3} \equiv$ $\mathrm{CNOT}_{B_{2} B_{1}} \mathrm{CNOT}_{B B_{2}} \mathrm{CNOT}_{B_{1} B}$. Then using two additional ebits $A_{3} C_{3}$ and $B_{4} C_{4}$, we swap $A_{3}$ with $A$ and $C_{4}$ with $C$. It then suffices to make the Bell measurements on $A_{1} A_{3}, B_{1} B_{3}, B_{2} B_{4}$, and $C_{2} C_{4}$. For correction gates, $B_{1} B_{3}$ will correct $A$ and $C_{2} C_{4}$ will correct $C$ as usual, $\left|\phi^{+}\right\rangle \rightarrow \mathbf{1},\left|\psi^{+}\right\rangle \rightarrow \sigma_{x},\left|\psi^{-}\right\rangle \rightarrow \sigma_{z} \sigma_{x}$, and $\left|\phi^{-}\right\rangle \rightarrow \sigma_{z}$, respectively. After these corrections, we then correct $A, B$ and $C$ with the result on $A_{1} A_{3}$ and $C_{2} C_{4}$ as shown in Table I.
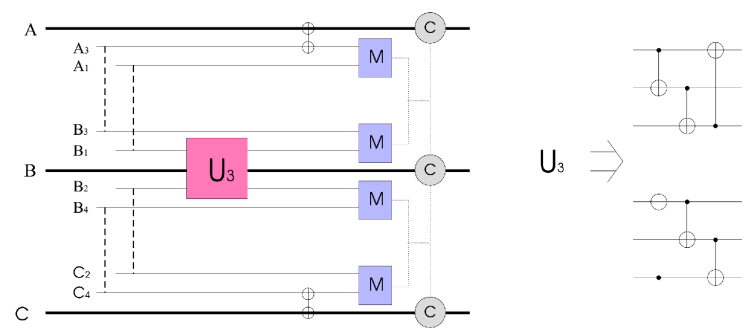

Fig. 3. Post-measurement nonlocal three-qubit gate. Here, four ebits are used: $A_{1} B_{1}, B_{2} C_{2}, A_{3} B_{3}$, and $B_{4} C_{4}$.

We consider another three-qubit nonlocal gate with $U_{3} \equiv \mathrm{CNOT}_{B C} \mathrm{CNOT}_{A B} \mathrm{CNOT}_{C A}$. We follow the same procedure as before and the measurement result on $B_{1} B_{3}$ and $B_{2} B_{4}$ corrects $A$ and $C$, respectively, as before. The correction gate for $A_{1} A_{3}$ and $B_{2} B_{4}$ is shown in Table II. 
TABLE I

Correction gates based on Bell measurements on $A_{1} A_{3}$ and $C_{2} C_{4}$. They are applied to qubits $A, B$, and $C$ for the post-measurement nonlocal three-qubit gate in Fig. 3 network for the case of $\mathrm{CNOT}_{C A} \mathrm{CNOT}_{B C} \mathrm{CNOT}_{A B}$.

\begin{tabular}{c|c|c|c|c}
\hline \hline & $\left|\phi^{+}\right\rangle_{C_{2} C_{4}}$ & $\left|\psi^{+}\right\rangle_{C_{2} C_{4}}$ & $\left|\psi^{-}\right\rangle_{C_{2} C_{4}}$ & $\left|\phi^{-}\right\rangle_{C_{2} C_{4}}$ \\
\hline$\left|\phi^{+}\right\rangle_{A_{1} A_{3}}$ & $\mathbf{1} \otimes \mathbf{1} \otimes \mathbf{1}$ & $\sigma_{x} \otimes \mathbf{1} \otimes \sigma_{x}$ & $\sigma_{x} \otimes \sigma_{z} \otimes \sigma_{z} \sigma_{x}$ & $\mathbf{1} \otimes \sigma_{z} \otimes \sigma_{z}$ \\
$\left|\psi^{+}\right\rangle_{A_{1} A_{3}}$ & $\mathbf{1} \otimes \sigma_{x} \otimes \sigma_{x}$ & $\sigma_{x} \otimes \sigma_{x} \otimes \mathbf{1}$ & $\sigma_{x} \otimes \sigma_{z} \sigma_{x} \otimes \sigma_{z}$ & $\mathbf{1} \otimes \sigma_{z} \sigma_{x} \otimes \sigma_{z} \sigma_{x}$ \\
$\left|\psi^{-}\right\rangle_{A_{1} A_{3}}$ & $\sigma_{z} \otimes \sigma_{x} \otimes \sigma_{z} \sigma_{x}$ & $\sigma_{z} \sigma_{x} \otimes \sigma_{x} \otimes \sigma_{z}$ & $\sigma_{z} \sigma_{x} \otimes \sigma_{z} \sigma_{x} \otimes \mathbf{1}$ & $\sigma_{z} \otimes \sigma_{z} \sigma_{x} \otimes \sigma_{x}$ \\
$\left|\phi^{-}\right\rangle_{A_{1} A_{3}}$ & $\sigma_{z} \otimes \mathbf{1} \otimes \sigma_{z}$ & $\sigma_{z} \sigma_{x} \otimes \mathbf{1} \otimes \sigma_{z} \sigma_{x}$ & $\sigma_{z} \sigma_{x} \otimes \sigma_{z} \otimes \sigma_{x}$ & $\sigma_{z} \otimes \sigma_{z} \otimes \mathbf{1}$
\end{tabular}

Correction gates based on Bell measurements on $A_{1} A_{3}$ and $C_{2} C_{4}$

TABLE II for $U_{3} \equiv \mathrm{CNOT}_{B C} \mathrm{CNOT}_{A B} \mathrm{CNOT}_{C A}$.

\begin{tabular}{c|c|c|c|c}
\hline \hline & $\left|\phi^{+}\right\rangle_{C_{2} C_{4}}$ & $\left|\psi^{+}\right\rangle_{C_{2} C_{4}}$ & $\left|\psi^{-}\right\rangle_{C_{2} C_{4}}$ & $\left|\phi^{-}\right\rangle_{C_{2} C_{4}}$ \\
\hline$\left|\phi^{+}\right\rangle_{A_{1} A_{3}}$ & $\mathbf{1} \otimes \mathbf{1} \otimes \mathbf{1}$ & $\sigma_{x} \otimes \sigma_{x} \otimes \mathbf{1}$ & $\sigma_{x} \otimes \sigma_{z} \sigma_{x} \otimes \sigma_{z}$ & $\mathbf{1} \otimes \sigma_{z} \otimes \sigma_{z}$ \\
$\left|\psi^{+}\right\rangle_{A_{1} A_{3}}$ & $\sigma_{x} \otimes \sigma_{x} \otimes \sigma_{x}$ & $\mathbf{1} \otimes \mathbf{1} \otimes \sigma_{x}$ & $\mathbf{1} \otimes \sigma_{z} \otimes \sigma_{z} \sigma_{x}$ & $\sigma_{x} \otimes \sigma_{z} \sigma_{x} \otimes \sigma_{z} \sigma_{x}$ \\
$\left|\psi^{-}\right\rangle_{A_{1} A_{3}}$ & $\sigma_{z} \sigma_{x} \otimes \sigma_{z} \sigma_{x} \otimes \sigma_{z} \sigma_{x}$ & $\sigma_{z} \otimes \sigma_{z} \otimes \sigma_{z} \sigma_{x}$ & $\sigma_{z} \otimes \mathbf{1} \otimes \sigma_{x}$ & $\sigma_{z} \sigma_{x} \otimes \sigma_{x} \otimes \sigma_{x}$ \\
$\left|\phi^{-}\right\rangle_{A_{1} A_{3}}$ & $\sigma_{z} \otimes \sigma_{z} \otimes \sigma_{z}$ & $\sigma_{z} \sigma_{x} \otimes \sigma_{z} \sigma_{x} \otimes \sigma_{z}$ & $\sigma_{z} \sigma_{x} \otimes \sigma_{x} \otimes \mathbf{1}$ & $\sigma_{z} \otimes \mathbf{1} \otimes \mathbf{1}$
\end{tabular}

\section{Conclusions}

We have studied some particular networks of CNOT gates for two and three qubits where the measurements were delayed until the end of the gates. In particular, we provided a two-qubit nonlocal gates for one, two, and three CNOT operations in which measurements are delayed. Post-measurement nonlocal three-qubit gates were also discussed using four ebit resources.

\section{References}

[1] J. Eisert, K. Jacobs, P. Papadopoulos, M.B. Plenio, Phys. Rev. A 62, 52317 (2000).

[2] D. Collins, N. Linden, S. Popescu, Phys. Rev. A 64, 032302 (2001).

[3] D. Gottesman, I. Chuang, Nature 402, 390 (2000).

[4] G.K. Brennen, D. Song, C.J. Williams, Phys. Rev. A 67, 050302(R) (2003). 